\title{
LA EDUCACIÓN COMPARADA MÁS ALLÁ DE LA REEC: ANÁLISIS BIBLIOMÉTRICO DE LA DISCIPLINA EN LAS CINCO REVISTAS ESPAÑOLAS AFINES DE MÁS IMPACTO ENTRE 1995 Y 2014
}

\section{Comparative Education beyond REEC: bibliometric}

analysis of the discipline in the five related Spanish journals with most impact between 1995 and 2014

Gabriel Álvarez López*

\section{RESUMEN}

Esta investigación consiste en un estudio bibliométrico que pretende analizar la producción científica en el ámbito de la Educación Comparada en cinco revistas especializadas españolas. El análisis nos permitirá identificar el estado actual de la disciplina en base a indicadores como el número de artículos publicados, temática, autores y procedencia (geográfica e institucional) o número de citas desde su publicación. Se han analizado 285 números de las revistas seleccionadas, extrayendo un total de 155 artículos de más de 300 autores procedentes de más de 15 países de 4 continentes. El artículo se cierra con una discusión acerca de la salud de la disciplina y los retos a los que se enfrenta desde el punto de vista de la producción científica.

\footnotetext{
* Universidad Autónoma de Madrid (España).
} 
PALABRAS CLAVE: Estudio Bibliométrico, Educación Comparada, Producción Científica.

\begin{abstract}
This investigation constists of a bibliometric study that aims to analyse the scientific production within the field of Comparative Education in five specialized Spanish journals. The analysis will allow us to identify the present state of the discipline, on the basis of factors such as the published articles, topics, authors, geographical or institutional background, or number or quotations from the said publication. There have been 285 issues analysed from the selected journals, and a total of 155 articles have been extracted out of more than 300 authors coming from over 15 countries and 4 continents. The article ends with a discussion upon the strength of the discipline as well as the challenges that it confronts from the point of view of the scientific production.
\end{abstract}

KEY WORDS: Bibliometric Study, Comparative Education, Scientific Production.

$* * * * *$

\title{
1. INTRODUCCIÓN
}

Con motivo del 20 aniversario de la publicación del primer número de la Revista Española de Educación Comparada resulta interesante analizar cuál ha sido la trayectoria de la disciplina más allá de una de sus revistas de referencia en castellano más significativas. Desde la creación de la REEC en 1995 hasta la actualidad, muchos han sido los espacios de publicación científica en los que investigadores y académicos afines a la Educación Comparada han encontrado para publicar sus trabajos e investigaciones. Este artículo presenta una investigación de corte descriptivo en la que se analiza el estado de las publicaciones sobre Educación Comparada a partir de los artículos aparecidos cinco revistas consideradas de prestigio en el panorama de las publicaciones académicas españolas: Revista de Educación, Revista Española de Pedagogía, Educación XX1, Profesorado. Revista de currículum y formación del profesorado y Bordón entre 1995 y 2014. De ellas se analizará este periodo de 20 años desde que se publicó el primer número de la REEC a través de una serie de parámetros como: temáticas tratadas, autores y sus procedencias, así como las citas que han tenido desde la publicación de los artículos hasta la actualidad. La elección de estos parámetros responde a la pretensión de presentar un escenario lo más completo posible del objetivo de este estudio.

En la última década no han sido pocos los artículos que han analizado otras dimensiones igualmente interesantes de la Educación Comparada como la cartografía académica (LÁZARO et al., 2014), las publicaciones fundamentalmente libros y artículos 
de revistas indexadas (EGIDO, 2014), los retos de la disciplina en un mundo globalizado (LÓPEZ, 2008; RAVENTÓS y PRATS, 2012; RAVENTÓS y GARCÍA, 2012) y su impacto (GARCÍA, 2012), los enfoques actuales y las indicaciones para la enseñanza y la investigación futura (LÁZARO, 2013) o los trabajos presentados en torno a las V Jornadas Nacionales de Docencia e Investigación en Educación Comparada sobre la situación de la Educación Comparada en los grados, los postgrados o la investigación ${ }^{1}$. Por otro lado, son igualmente pertinentes los artículos que se publicaron con motivo del $10^{\circ}$ aniversario de la REEC y trataron temas como la trayectoria de la disciplina en España (GARCÍA GARRIDO, 2005), la situación y los desafíos de la Educación Comparada en América Latina (FERNÁNDEZ LAMARRA, MOLLIS y DONO, 2005), o el estudio sobre la ocupación y el uso que la disciplina hizo de los nuevos espacios virtuales (webs, revistas y listas de distribución) de Luis María Naya (2005). Especial relevancia para este análisis tiene el estudio publicado por María Jesús Martínez Usarralde y Javier M. Valle (2005) en el que realizan una mirada en prospectiva sobre los 10 años de la Revista y presentan un estudio bibliométrico de 1995 a 2004 centrándose en las temáticas, la naturaleza de los estudios y las unidades de comparación utilizadas.

Este artículo se suma a la trayectoria abierta por todos estos trabajos previos en un modesto intento de actualizarlos aprovechando la efeméride del 20 aniversario, aportando una visión más amplia en el tiempo e introduciendo parámetros hasta ahora novedosos como el impacto de la producción científica.

\section{MARCO TEÓRICO}

Los análisis o estudios bibliométricos están sufriendo un crecimiento exponencial en la literatura científica española. Podemos considerar este crecimiento en base al desarrollo del Espacio Europeo de Educación Superior por un lado, y por otro lado como consecuencia de la implantación de los procesos de acreditación llevado a cabo por la Agencia Nacional de Evaluación de la Calidad y Acreditación (ANECA). En este proceso acreditativo, el apartado más importante dentro de la investigación es la publicación de artículos en revistas científicas (ANECA, 2007, 2008). Por otra parte, la Comisión Nacional Evaluadora de la Actividad Investigadora (CNEAI), para la concesión de los tramos de investigación o sexenios, también tiene en cuenta los artículos científicos (RUIZ-PÉREZ et al., 2010). Así pues, los artículos científicos se han convertido en los protagonistas absolutos, muy por encima de los libros, en lo que a la evaluación de la producción científica de los profesores e investigadores españoles se refiere.

Este auge de los artículos ha venido acompañado por otro incremento considerable como es el sufrido por las revistas científicas del ámbito educativo. En un reciente artículo

\footnotetext{
${ }^{1}$ Disponibles en https://canal.uned.es/serial/index/id/1013
} 
(RUIZ-CORBELLA et al., 2014) se analiza esta evolución y se justifica en base a dos grupos de argumentos. Por un lado, como ya hemos comentado anteriormente, por la necesidad de publicar producciones "con impacto" y, por otro lado, por el desarrollo de políticas de promoción y autonomía de las universidades españolas. Ambos aspectos son determinantes para entender la gran evolución de estas revistas en España, la gran mayoría vinculadas a universidades públicas. Aunque el auge vino en los 80 , el fortalecimiento y el impacto de estas revistas no llegó hasta finales de los 2000, cuando en 2010 Revista de Educación y Revista Española de Pedagogía consiguen entrar en el prestigioso Social Sciences Citation Index del Journal Citation Reports (JCR) de Thompson Reuters. De 2010 a 2013 (última actualización del índice JCR) el crecimiento ha sido considerable y actualmente siete revistas científicas españolas del ámbito educativo están dentro de él.

Con este panorama de partida, los estudios bibliométricos se justifican por la necesidad de estudiar qué se está publicando en las revistas científicas, qué impacto están teniendo los artículos y cómo se están desarrollando las diferentes disciplinas. En este sentido, en los últimos años, en el ámbito educativo se han publicado estudios o análisis bibliométricos de diferentes tipos: comparaciones entre revistas (FUENTES et al., 2012), estudios de una sola revista (CARPALLO y BURGOS, 2008; GRANADOS et al., 2011; ZYCH, 2011; GÓMEZ GARCÍA et al., 2012; LÓPEZ et al., 2015), o sobre la producción científica de disciplinas concretas (NIKLEVA y CORTINA, 2014). En este último grupo estaría ubicado el estudio que se está presentando.

\section{METODOLOGÍA}

\subsection{Unidad de análisis}

La unidad de análisis serán los artículos científicos publicados en las cinco revistas con mayor impacto y cercanas a las disciplinas afines a la Educación Comparada en España desde 1995 hasta 2014, sobre investigaciones que apliquen el método comparado en alguna de sus fases (GARCÍA GARRIDO, 1996), o que se sirvan de la comparación para resaltar convergencias y divergencias en sus elementos de análisis. Han quedado excluidas del estudio introducciones, editoriales y recensiones.

Se han revisado 285 ejemplares de las revistas seleccionadas, extrayendo 155 artículos de 301 autores y autoras de más de 15 países de 4 continentes.

\subsection{Material que es objeto de nuestra muestra}

Para la selección de las revistas se tuvieron en cuenta diferentes índices de impacto y de calidad de la revista (Tabla 1). En primer lugar, el índice de impacto de mayor presencia e importancia internacional, desarrollado por Thompson Reuters, el Journal of 
Citation Report del año 2013. En segundo lugar, se tuvo en cuenta el índice de la Clasificación Integrada de Revistas Científicas (CIRC) desarrollado por Torres - Salinas et al. (2010), promovido por el Grupo de Evaluación de Publicaciones Científicas (EPUC) y asumido en la actualidad por el grupo EC3 de la Universidad de Granada. En tercer lugar, se ha tenido también en cuenta el Sello de Calidad que otorga la Fundación Española para la Ciencia y la Tecnología (FECYT), de Delgado et al. (2006), actualizado por última vez en Septiembre del año 2014; para este último criterio se ha valorado la posesión o no posesión del sello en la última actualización.

Dentro de estos criterios, se seleccionaron aquellas revistas con más cercanía a las disciplinas afines a la Educación Comparada, eliminando por lo tanto, Cultura y Educación, Comunicar, Enseñanza de las ciencias y Porta Linguarum. Por lo tanto, las revistas finalmente seleccionadas fueron (Tabla 2) Revista de Educación, Revista Española de Pedagogía, Educación XX1, Profesorado. Revista de Currículum y Formación del Profesorado (en adelante "Revista Profesorado") y la revista Bordón.

Tabla 1. Las 10 revistas de mayor impacto en el ámbito educativo

\begin{tabular}{|l|c|c|c|}
\hline NOMBRE & JCR 2013 & CIRC 2012 & SELLO FECYT \\
\hline Cultura y Educación & 0.375 & GRUPO A & SI \\
\hline Comunicar & 0.350 & GRUPO A & SI \\
\hline Revista de Educación & 0.201 & GRUPO A & SI \\
\hline Enseñanza de las ciencias & 0.103 & GRUPO A & NO \\
\hline Revista Española de Pedagogía & 0.093 & GRUPO A & NO \\
\hline Porta Linguarum & 0.058 & GRUPO A & SI \\
\hline Educación XX1 & 0.020 & GRUPO A & SI \\
\hline Profesorado & - & GRUPO B & SI \\
\hline Bordón & - & GRUPO B & SI \\
\hline Aula Abierta & - & GRUPO B & NO \\
\hline
\end{tabular}

* Fuente: elaboración propia.

En este punto se debe señalar que para los casos de las revistas Profesorado y Educación XX1 únicamente se han podido tener en cuenta las publicaciones desde 1997 y 1998 respectivamente, porque han sido las fechas de creación de ambas revistas. Por lo tanto, el periodo de 1995 a 1996 estará representado únicamente por tres de las cinco revistas. Además, en el caso de la Revista Profesorado los artículos seleccionados correspondientes a los periodos 1997 - 1999 y 2009 - 2014, no estaban accesibles a texto completo y se han seleccionado en función del resumen. Esto también ha pasado con la revista Bordón cuyos artículos se han seleccionado en función del resumen que facilitaba la base de datos Dialnet de la Universidad de la Rioja, a la cual te reconduce la propia página web de la revista Bordón.

Tabla 2. Datos generales de las revistas seleccionadas 


\begin{tabular}{|l|c|c|c|c|c|}
\hline & $\begin{array}{c}\text { Revista de } \\
\text { Educación }\end{array}$ & $\begin{array}{c}\text { Revista } \\
\text { Española } \\
\text { de } \\
\text { Pedagogía }\end{array}$ & $\begin{array}{c}\text { Educación } \\
\text { XX1 }\end{array}$ & Profesorado & Bordón \\
\hline $\begin{array}{l}\text { Periodo en } \\
\text { activo de la } \\
\text { revista }\end{array}$ & $\begin{array}{c}\text { Desde } \\
1940\end{array}$ & $\begin{array}{c}\text { Desde } \\
1943\end{array}$ & $\begin{array}{c}\text { Desde } \\
1998\end{array}$ & $\begin{array}{c}\text { Desde } \\
1997\end{array}$ & $\begin{array}{c}\text { Desde } \\
1949\end{array}$ \\
\hline $\begin{array}{l}\text { Ejemplares por } \\
\text { año durante el } \\
\text { periodo } \\
\text { estudiado }\end{array}$ & $\begin{array}{l}3(1995-2013) \\
4(2014)+\end{array}$ & 3 & $\begin{array}{l}1(1998- \\
2009) \\
\text { extraordinarios }\end{array}$ & $\begin{array}{l}2(19010-) \\
2006) \\
3(2007-)\end{array}$ & $\begin{array}{l}4 \\
5 \\
(2005 / 6)\end{array}$ \\
\hline $\begin{array}{l}\mathbf{N}^{\mathbf{0}} \text { de } \\
\text { ejemplares } \\
\text { revisados }\end{array}$ & 77 & 60 & 22 & 44 & 82 \\
\hline $\begin{array}{l}\mathbf{N}^{\mathbf{0}} \text { de artículos } \\
\text { seleccionados }\end{array}$ & 84 & 16 & 9 & 26 & 20 \\
\hline
\end{tabular}

* Fuente: elaboración propia.

\section{$\underline{\text { 3.3. Diseño y procedimiento }}$}

Se trata de un estudio descriptivo mediante análisis de documentos, según la clasificación propuesta por Montero y León (2007), redactado siguiendo las normas propuestas por Ramos - Álvarez, Moreno - Fernández, Valdés - Conroy y Catena (2008). En la selección de los artículos no se tuvieron en cuenta ni introducciones, ni editoriales, ni recensiones publicadas en las revistas analizadas.

Una vez se definió la unidad de análisis, se procedió a la recogida de datos para su posterior análisis. En primer lugar, se recogieron los datos de las temáticas según el tema de cada artículo y se categorizaron en 20 temáticas finales en función de su afinidad. Seguidamente fueron los datos de los autores y su procedencia, lo que he llamado "Geografía de la Educación Comparada". Finalmente se recogieron las citas de cada artículo, para lo que se realizó una búsqueda a través de Google Scholar la última semana de Febrero de 2015. Todos estos datos se volcaron en hojas de Microsoft Excel para su análisis, teniendo en cuenta indicadores cualitativos y cuantitativos. Los indicadores cualitativos seleccionados fueron: revista, año, temática, autor/es, procedencia (país, comunidad autónoma si es en España) e institución a la que pertenecen los autores. En cuanto a los indicadores cuantitativos seleccionados fueron los siguientes: número de artículos por revista, número de autores por artículo y número de citas de cada artículo.

\section{RESULTADOS}

Para una lectura más fluida de los resultados, se presentan agrupados en diferentes subapartados. En primer lugar, los resultados más genéricos referentes a número de 
artículos, artículos por revista y artículos por años. En segundo lugar encontraremos el subapartado de autores en el que podremos analizar el número de autores por artículo, los autores más prolíficos. Las temáticas se encuentran en el tercer subapartado, y en este caso podremos analizar las temáticas seleccionadas, el número de artículos por temática, el porcentaje de temáticas por el total de artículos, las temáticas más presentes en cada revista y cómo han ido variando las temáticas a lo largo de los años. El siguiente subapartado, el cuarto, hará referencia a las citas, y se presentan los resultados del total de citas por revista, las citas por año y revista, los artículos más citados, así como el número de citas en función de los años. Por último, el quinto subapartado hace referencia a la geografía de la comparada, es decir, el tipo de organismos a los que pertenecen los autores, los países de procedencia, las comunidades autónomas (en el caso de los autores españoles) y las universidades con mayor número de artículos publicados por sus docentes.

La exhaustividad en la presentación de los resultados no tiene otra razón que la de hacer un estudio lo más amplio posible sobre el estado de la Educación Comparada, queriendo abarcar todos los aspectos relevantes en lo que ha producción científica se refiere.

\subsection{Resultados generales}

La presencia de artículos de educación comparada en las revistas analizadas es bastante desigual en dos sentidos. En primer lugar, como podemos ver en el Gráfico 1, el número de artículos por revista es muy variable, siendo Revista de Educación la que más artículos aporta. Además, como podemos ver en la Tabla 1, únicamente en 5 ocasiones ha habido años en los que se hayan encontrado más de 5 artículos en una misma publicación (2004, 2005, 2006 y 2008 en Revista de Educación y 2010 en Revista Profesorado). Es siempre Revista de Educación quien impulsa las estadísticas en lo que a número de artículos publicados se refiere, salvo en los casos de 2009 que fue Revista Española de Pedagogía quien publicó más artículos, y 2013 que fue Revista Educación XX1.

Tabla 3. Relación de artículos por revistas y años de publicación

\begin{tabular}{|c|c|c|c|c|c|c|c|c|c|c|c|c|c|c|c|c|c|c|c|c|}
\hline & $\mathscr{2}$ & ڤั & $\hat{\sigma}$ & $\stackrel{2}{2}$ & $\hat{\sigma}$ & ปิ่ & ฮิ่ & ฮิ & ๕ิેి & ఫ્ડ & 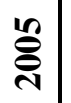 & ֻัڤે & હิે & 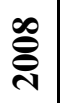 & ڤે & 을 & $\overline{\bar{\Omega}}$ & 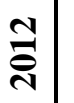 & $\stackrel{m}{\bar{\sim}}$ & 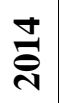 \\
\hline $\begin{array}{l}\text { Revista de } \\
\text { Educación }\end{array}$ & 2 & 1 & 4 & 5 & 2 & 5 & 4 & 5 & 4 & 12 & 6 & 8 & 4 & 7 & 1 & 4 & 4 & 2 & 1 & 3 \\
\hline $\begin{array}{l}\text { Revista } \\
\text { Española de } \\
\text { Pedagogía } \\
\end{array}$ & 1 & 1 & 0 & 1 & 0 & 0 & 2 & 1 & 1 & 1 & 1 & 0 & 1 & 1 & 2 & 1 & 2 & 0 & 0 & 0 \\
\hline $\begin{array}{l}\text { Educación } \\
\text { XX1 }\end{array}$ & & & & 0 & 0 & 0 & 0 & 0 & 0 & 0 & 0 & 1 & 0 & 1 & 1 & 1 & 1 & 0 & 2 & 2 \\
\hline
\end{tabular}




\begin{tabular}{|c|c|c|c|c|c|c|c|c|c|c|c|c|c|c|c|c|c|c|c|c|}
\hline $\begin{array}{l}\text { Revista } \\
\text { Profesorado }\end{array}$ & & & 0 & 0 & 1 & 0 & 0 & 0 & 0 & 1 & 1 & 3 & 2 & 2 & 0 & 11 & 2 & 1 & 1 & 1 \\
\hline $\begin{array}{l}\text { Revista } \\
\text { Bordón }\end{array}$ & 1 & 0 & 1 & 2 & 0 & 1 & 0 & 0 & 1 & 1 & 1 & 2 & 0 & 2 & 1 & 3 & 1 & 1 & 0 & 2 \\
\hline
\end{tabular}

* Fuente: elaboración propia.

** Nota: se recuerda que para las revistas Educación XX1 y Profesorado el periodo de análisis es desde su creación hasta 2014.

Para el caso de la revista Profesorado es interesante apuntar que de los 11 artículos del año 2010, 10 se deben a un monográfico sobre los planes de estudio de la formación inicial del profesorado de Música en América Latina y Europa, estableciendo en la mayoría de los artículos comparaciones entre países.

Gráfico 1. Número de artículos por revistas

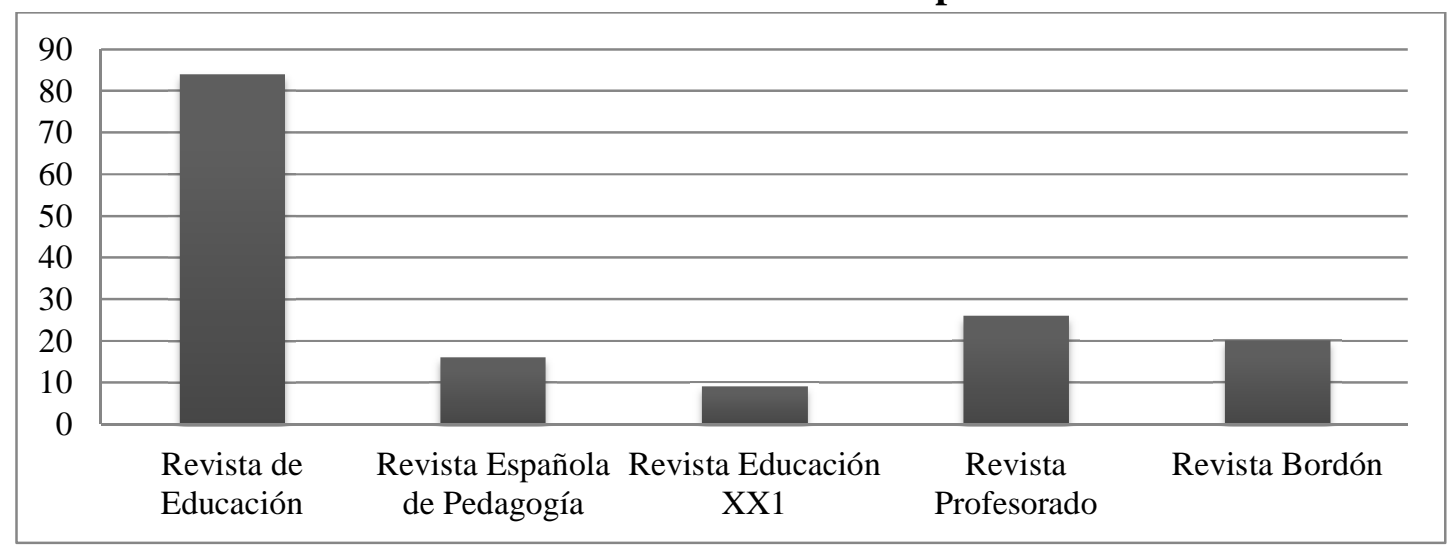

* Fuente: elaboración propia.

La otra desigualdad en la presencia de artículos la encontramos en el periodo analizado (Gráfico 2). En total, teniendo en cuenta las cinco revistas, únicamente se encuentran 10 artículos o más en un año en los casos de 2004, 2006, 2008, 2010 y 2011. Sin embargo, es destacable que todos los años se han publicado un mínimo 3 artículos, salvo en el caso de 1996 que se publicaron 2. Además, sólo en 1995, 1996, 1999, 2012 y 2013 se han publicado menos de 5 artículos entre todas las revistas analizadas. El año que más artículos se publicaron fue el 2010, impulsado por el monográfico que ya hemos apuntado de la revista Profesorado.

\section{Gráfico 2. Artículos por años}




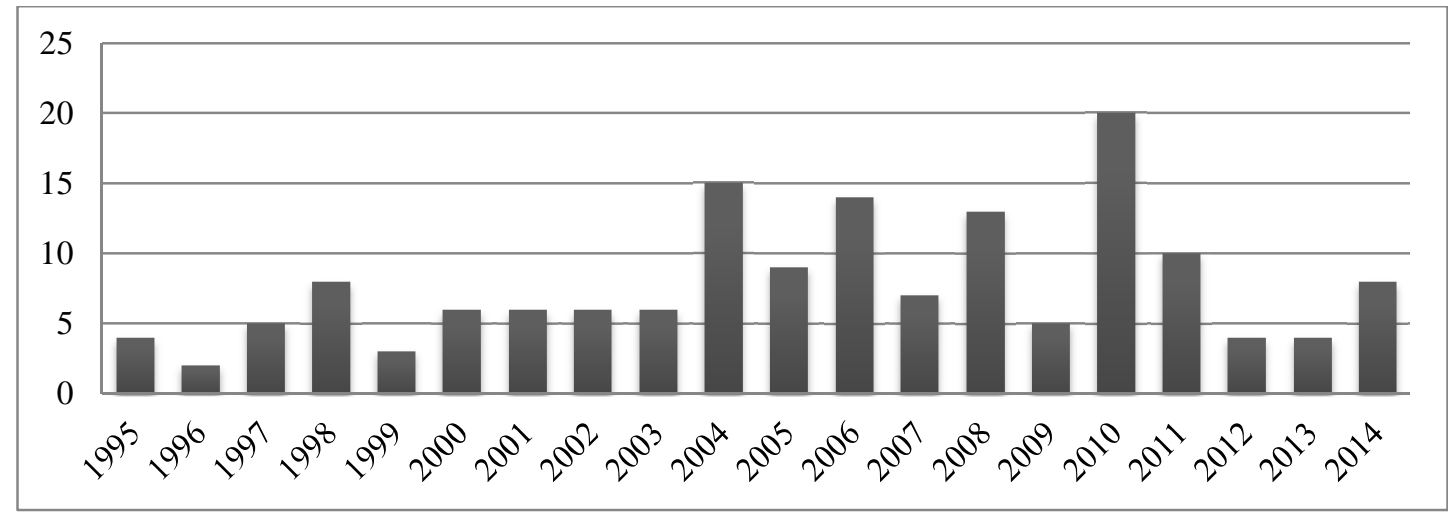

* Fuente: elaboración propia.

En el gráfico 3 se resume de forma gráfica las dos desigualdades que hemos ido tratando en este apartado y se observa perfectamente como es Revista de Educación la que más artículos afines a la disciplina publica y cómo el monográfico de Profesorado en 2010 contribuyó notablemente a la presencia del método comparado.

\section{Gráfico 3. Evolución de la producción científica por revista y años}

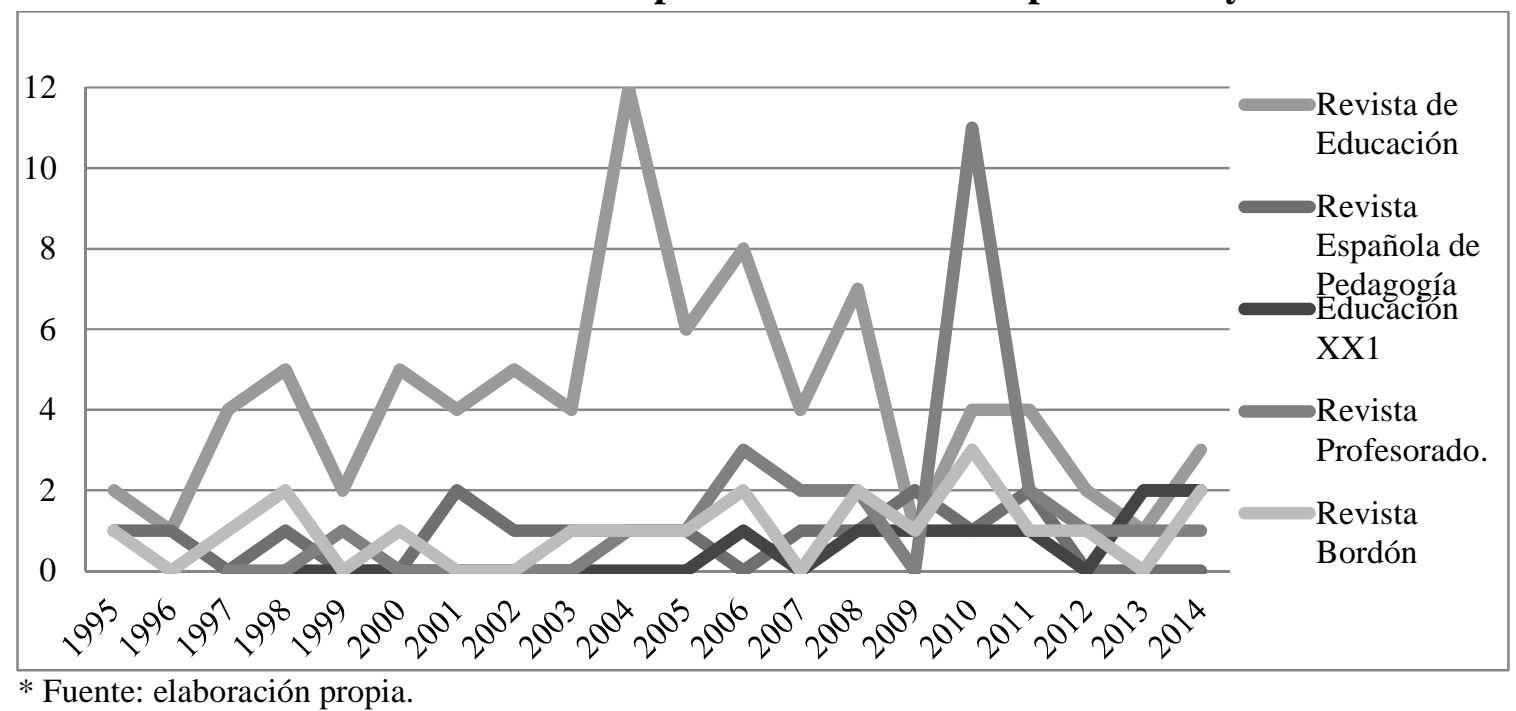

\subsection{Autores}

Los resultados para el caso de los autores muestran que la mayoría de artículos seleccionados han sido escritos por un solo autor (79), siendo incluso superior a los artículos escritos por más de un autor (76). Se ha dado un único caso en el que no ha sido posible determinar el número total de autores porque el índice de autoría de ese artículo explicitaba un autor y seguidamente nombraba a los grupos de investigación implicados en la investigación, pero no daba sus nombres completos, así que se ha optado por etiquetarlo 
como "indeterminado", aunque el autor principal sí que está en el tratamiento del resto de datos.

\section{Gráfico 4. Número de autores por artículo}

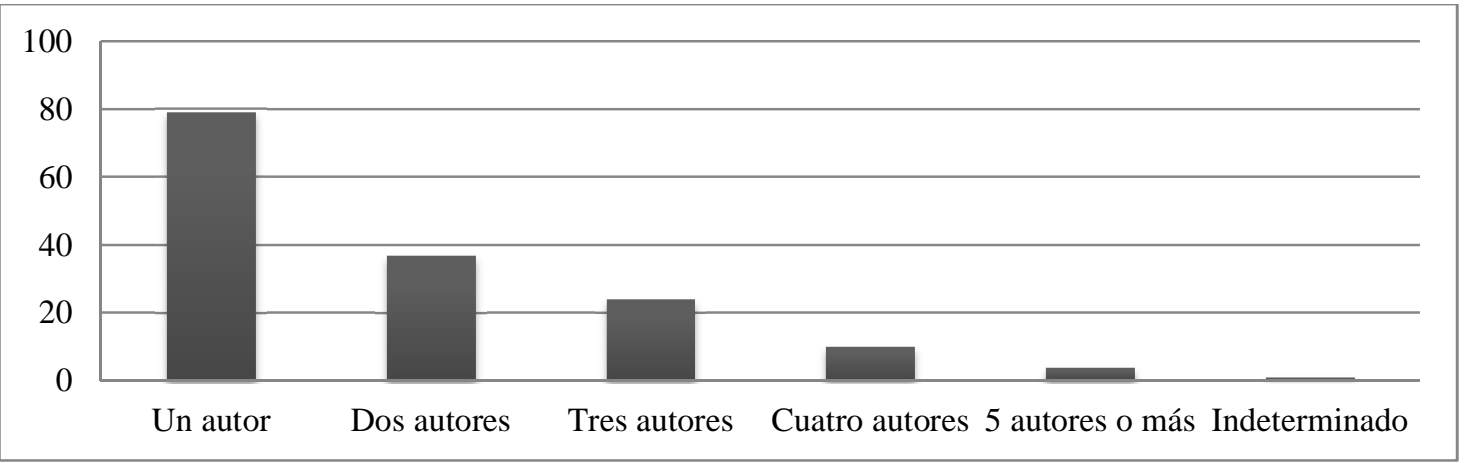

* Fuente: elaboración propia.

En la Tabla 4 los autores más prolíficos con más de un artículo firmado en solitario o en coautoría por orden alfabético de nombre y apellidos. También podemos observar como la mayoría de casos de autores presentes en la tabla tienen los artículos bastante cercanos en el tiempo, esto se puede deber a que hayan hecho alguna investigación relevante y la hayan presentado en dos artículos diferentes, en ocasiones hasta en la misma revista.

Tabla 4. Autores más prolíficos

\begin{tabular}{|l|l|l|l|l|}
\hline \multicolumn{1}{|c|}{ REVISTA } & AÑO & \multicolumn{1}{|c|}{$\begin{array}{c}\text { NOMBRE Y } \\
\text { APELLIDOS }\end{array}$} & \multicolumn{1}{|c|}{ UNIVERSIDAD } & PAÍS \\
\hline Bordón & 1998 & Vicente Llorent & $\begin{array}{l}\text { Universidad de } \\
\text { Sevilla }\end{array}$ & España \\
\hline $\begin{array}{l}\text { Revista de } \\
\text { Educación }\end{array}$ & 2004 & Vicente Llorent & $\begin{array}{l}\text { Universidad de } \\
\text { Sevilla }\end{array}$ & España \\
\hline Educación XX1 & 2009 & Vicente Llorent & $\begin{array}{l}\text { Universidad de } \\
\text { Sevilla }\end{array}$ & España \\
\hline $\begin{array}{l}\text { Revista de } \\
\text { Educación }\end{array}$ & 2014 & Arturo Galán & UNED & España \\
\hline Educación XX1 & 2014 & Arturo Galán & UNED & España \\
\hline Profesorado & 2010 & $\begin{array}{l}\text { Edith Cisneros- } \\
\text { Cohenour }\end{array}$ & $\begin{array}{l}\text { Universidad } \\
\text { Autónoma de } \\
\text { Yucatán }\end{array}$ & México \\
\hline Profesorado & 2010 & $\begin{array}{l}\text { Edith Cisneros- } \\
\text { Cohenour }\end{array}$ & $\begin{array}{l}\text { Universidad } \\
\text { Autónoma de } \\
\text { Yucatán }\end{array}$ & México \\
\hline $\begin{array}{l}\text { Revista de } \\
\text { Educación }\end{array}$ & 2008 & Esther Belvis & $\begin{array}{l}\text { Universidad } \\
\text { Autónoma de } \\
\text { Barcelona }\end{array}$ & España \\
\hline
\end{tabular}




\begin{tabular}{|c|c|c|c|c|}
\hline $\begin{array}{l}\text { Revista de } \\
\text { Educación }\end{array}$ & 2008 & Esther Belvis & - & - \\
\hline $\begin{array}{l}\text { Revista Española de } \\
\text { Pedagogía }\end{array}$ & 2002 & Felipe Martínez & $\begin{array}{l}\text { Universidad } \\
\text { Autónoma de } \\
\text { Aguascalientes }\end{array}$ & México \\
\hline $\begin{array}{l}\text { Revista de } \\
\text { Educación }\end{array}$ & 2006 & Felipe Martínez & $\begin{array}{l}\text { Instituto Nacional } \\
\text { de Evaluación de la } \\
\text { Educación }\end{array}$ & México \\
\hline Educación XX1 & 2010 & Inmaculada Egido & $\begin{array}{l}\text { Universidad } \\
\text { Complutense de } \\
\text { Madrid }\end{array}$ & España \\
\hline Educación XX1 & 2014 & Inmaculada Egido & $\begin{array}{l}\text { Universidad } \\
\text { Complutense de } \\
\text { Madrid }\end{array}$ & España \\
\hline Profesorado & 2007 & Jesús Viciana & $\begin{array}{l}\text { Universidad de } \\
\text { Huelva }\end{array}$ & España \\
\hline Profesorado & 2013 & Jesús Viciana & $\begin{array}{l}\text { Universidad de } \\
\text { Granada }\end{array}$ & España \\
\hline $\begin{array}{l}\text { Revista Española de } \\
\text { Pedagogía }\end{array}$ & 2009 & Joaquín Gairín & $\begin{array}{l}\text { Universidad } \\
\text { Autónoma de } \\
\text { Barcelona }\end{array}$ & España \\
\hline Profesorado & 2010 & Joaquín Gairín & $\begin{array}{l}\text { Universidad } \\
\text { Autónoma de } \\
\text { Barcelona }\end{array}$ & España \\
\hline $\begin{array}{l}\text { Revista de } \\
\text { Educación }\end{array}$ & 2003 & José Ginés Mora & $\begin{array}{l}\text { Universidad de } \\
\text { Valencia }\end{array}$ & España \\
\hline $\begin{array}{l}\text { Revista de } \\
\text { Educación }\end{array}$ & 2012 & José Ginés Mora & $\begin{array}{l}\text { Universidad de } \\
\text { Londres }\end{array}$ & $\begin{array}{l}\text { Reino } \\
\text { Unido } \\
\end{array}$ \\
\hline $\begin{array}{l}\text { Revista de } \\
\text { Educación }\end{array}$ & 2011 & José Joaquín Bruner & $\begin{array}{l}\text { Universidad Diego } \\
\text { Portales }\end{array}$ & Chile \\
\hline Educación XX1 & 2014 & José Joaquín Bruner & $\begin{array}{l}\text { Universidad Diego } \\
\text { Portales }\end{array}$ & Chile \\
\hline Bordón & 2004 & $\begin{array}{l}\text { José Luis García } \\
\text { Garrido }\end{array}$ & UNED & España \\
\hline Bordón & 2006 & $\begin{array}{l}\text { José Luis García } \\
\text { Garrido } \\
\end{array}$ & UNED & España \\
\hline $\begin{array}{l}\text { Revista Española de } \\
\text { Pedagogía }\end{array}$ & 2009 & José Luís Muñoz & $\begin{array}{l}\text { Universidad } \\
\text { Autónoma de } \\
\text { Barcelona }\end{array}$ & España \\
\hline Profesorado & 2010 & José Luís Muñoz & $\begin{array}{l}\text { Universidad } \\
\text { Autónoma de } \\
\text { Barcelona } \\
\end{array}$ & España \\
\hline Bordón & 2005 & Juan Antonio López & $\begin{array}{l}\text { Universidad } \\
\text { Autónoma de } \\
\text { Barcelona } \\
\end{array}$ & España \\
\hline Bordón & 2010 & Juan Antonio López & Universidad de & España \\
\hline
\end{tabular}




\begin{tabular}{|c|c|c|c|c|}
\hline & & & Granada & \\
\hline Bordón & 2008 & Karmele Buján & $\begin{array}{l}\text { Universidad del } \\
\text { País Vasco }\end{array}$ & España \\
\hline Bordón & 2012 & Karmele Buján & $\begin{array}{l}\text { Universidad del } \\
\text { País Vasco }\end{array}$ & España \\
\hline Bordón & 2009 & Luis María Naya & $\begin{array}{l}\text { Universidad del } \\
\text { País Vasco }\end{array}$ & España \\
\hline Educación XX1 & 2011 & Luis María Naya & $\begin{array}{l}\text { Universidad de País } \\
\text { Vasco }\end{array}$ & España \\
\hline $\begin{array}{l}\text { Revista Española de } \\
\text { Pedagogía }\end{array}$ & 2001 & $\begin{array}{l}\text { María Jesús Martínez } \\
\text { Usarralde }\end{array}$ & $\begin{array}{l}\text { Universidad de } \\
\text { Valencia }\end{array}$ & España \\
\hline $\begin{array}{l}\text { Revista de } \\
\text { Educación }\end{array}$ & 2008 & $\begin{array}{l}\text { María Jesús Martínez } \\
\text { Usarralde }\end{array}$ & $\begin{array}{l}\text { Universidad de } \\
\text { Valencia }\end{array}$ & España \\
\hline $\begin{array}{l}\text { Revista de } \\
\text { Educación }\end{array}$ & 2013 & María Victoria López & $\begin{array}{l}\text { Universidad de } \\
\text { Granada }\end{array}$ & España \\
\hline $\begin{array}{l}\text { Revista de } \\
\text { Educación }\end{array}$ & 2014 & María Victoria López & $\begin{array}{l}\text { Universidad de } \\
\text { Granada }\end{array}$ & España \\
\hline $\begin{array}{l}\text { Revista de } \\
\text { Educación }\end{array}$ & 2008 & María Victoria Moreno & \begin{tabular}{|l} 
Universidad \\
Autónoma de \\
Barcelona \\
\end{tabular} & España \\
\hline $\begin{array}{l}\text { Revista de } \\
\text { Educación }\end{array}$ & 2008 & María Victoria Moreno & - & - \\
\hline Bordón & 2009 & Paulí Dávila & $\begin{array}{l}\text { Universidad del } \\
\text { País Vasco }\end{array}$ & España \\
\hline Educación XX1 & 2011 & Paulí Dávila & $\begin{array}{l}\text { Universidad de País } \\
\text { Vasco }\end{array}$ & España \\
\hline $\begin{array}{l}\text { Revista de } \\
\text { Educación }\end{array}$ & 2011 & Pedro Aramendi & $\begin{array}{l}\text { Universidad del } \\
\text { País Vasco }\end{array}$ & España \\
\hline Bordón & 2012 & Pedro Aramendi & $\begin{array}{l}\text { Universidad del } \\
\text { País Vasco } \\
\end{array}$ & España \\
\hline $\begin{array}{l}\text { Revista de } \\
\text { Educación }\end{array}$ & 2008 & Pilar Pineda & $\begin{array}{l}\text { Universidad } \\
\text { Autónoma de } \\
\text { Barcelona } \\
\end{array}$ & España \\
\hline $\begin{array}{l}\text { Revista de } \\
\text { Educación }\end{array}$ & 2008 & Pilar Pineda & $\begin{array}{l}\text { Universidad } \\
\text { Autónoma de } \\
\text { Barcelona }\end{array}$ & España \\
\hline $\begin{array}{l}\text { Revista de } \\
\text { Educación }\end{array}$ & 2008 & Rosa Bruno-Jofré & Queen's University & Canadá \\
\hline Bordón & 2010 & Rosa Bruno-Jofré & Queen's University & Canadá \\
\hline
\end{tabular}

* Fuente: elaboración propia.

\subsection{Temáticas}

Para este subapartado ha sido necesario hacer un proceso de agrupamiento de las temáticas. En un primer momento, se fueron recogiendo los temas de cada artículo en 
función de las palabras clave y de los temas que se estuvieran tratando; se recogieron 81 temas diferentes. Estos temas se agruparon en función de la afinidad de la temática, quedando finalmente un conjunto de 20, más manejables estadísticamente y con mayor relevancia para el tratamiento de los datos. No fue posible agrupar 7 de las temáticas finales: TICs, libros de texto, género, escuela rural, educación para la salud, comprensividad y abandono escolar temprano. El resto son el resultado de la agrupación de otros temas. Por ejemplo, la temática de "Educación superior" es el resultado de la unión de "acceso a la universidad", "rendimiento de los estudiantes universitarios", "competencias egresados universitarios", "internacionalización y globalización de la educación superior", "planes de estudio universitarios", "EEES", "movilidad universitaria", "gobierno corporativo de las universidades", "análisis de las titulaciones sobre Educación", "universidad pública en América Latina", "universidades populares", "apoyo al estudiante universitario", "evaluación de títulos de postgrado", "valoración de títulos" y "formación previa de los universitarios".

Tabla 5. Número de artículos por temática

\begin{tabular}{|l|c|}
\hline \multicolumn{1}{|c|}{ Temáticas } & $\mathbf{N}^{\mathbf{0}}$ de artículos \\
\hline Profesorado & 29 \\
\hline Educación superior & 27 \\
\hline Política educativa & 19 \\
\hline Métodos, investigación e innovación educativa & 12 \\
\hline Clima y convivencia escolar & 9 \\
\hline Centros y dirección escolar & 8 \\
\hline Evaluación y rendimiento & 7 \\
\hline Apoyo educativo y atención a la diversidad & 7 \\
\hline TICS & 6 \\
\hline Competencias clave - básicas & 6 \\
\hline Integración social & 5 \\
\hline Didáctica de las lenguas & 4 \\
\hline Libros de texto & 3 \\
\hline Interculturalidad y Desarrollo & 3 \\
\hline Formación profesional y Educación de adultos & 3 \\
\hline Género & 2 \\
\hline Escuela rural & 2 \\
\hline Educación para la salud & 1 \\
\hline Comprensividad & 1 \\
\hline Abandono escolar temprano & 1 \\
\hline
\end{tabular}

* Fuente: elaboración propia

Como podemos observar en el Gráfico 5, las temáticas más comunes han sido "Profesorado", "Educación Superior" y "Política Educativa", siempre por encima del 10\% del total de artículos. Esta mayor presencia es debida en gran parte a que son las temáticas 
más comunes de los artículos presentes en la Revista de Educación (Gráfico 6) que, como ya hemos visto, es la revista que ha tenido más artículos seleccionados.

\section{Gráfico 5. Porcentajes de temáticas en el total de artículos}

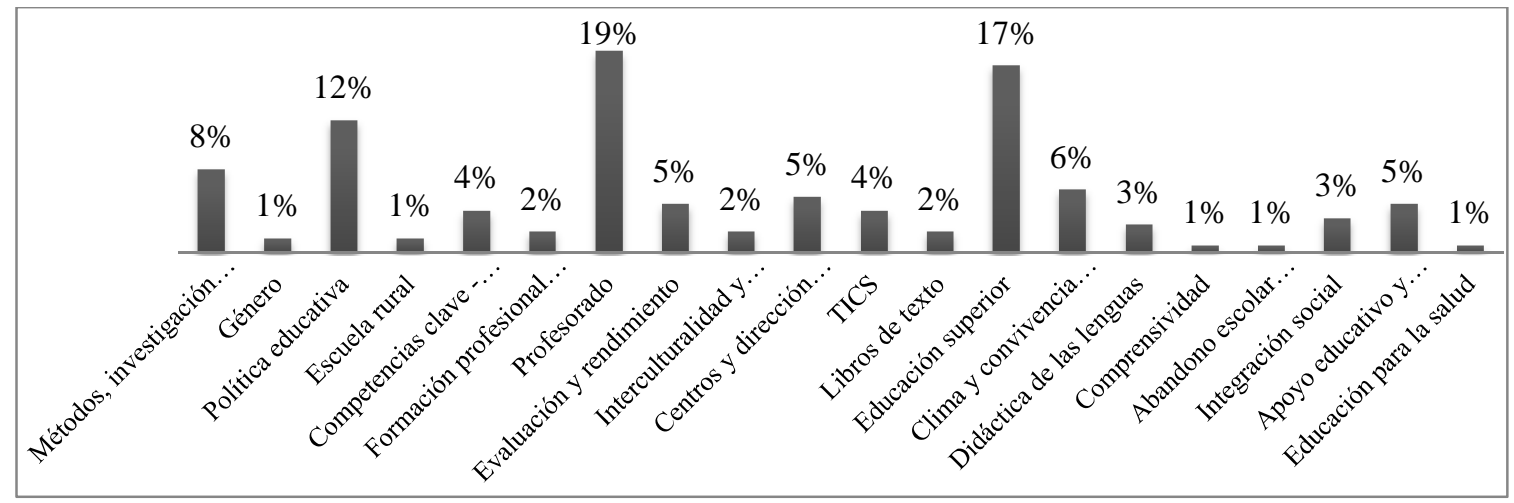

* Fuente: elaboración propia.

Llama la atención la presencia de "Métodos, investigación e innovación educativa" con el 8\%, pero recordemos que no se están analizando artículos que versen sobre el estado de la Comparada en España o el sobre método comparado, sino que hagan uso de él en alguna de las fases de la investigación que presentan o el análisis que realicen en el propio artículo; de esta forma, esta temática agrupa artículos que han establecido comparaciones entre métodos de investigación, metodologías didácticas, resultados de innovaciones educativas, etc.

Gráfico 6. Número de artículos por temática en la Revista de Educación

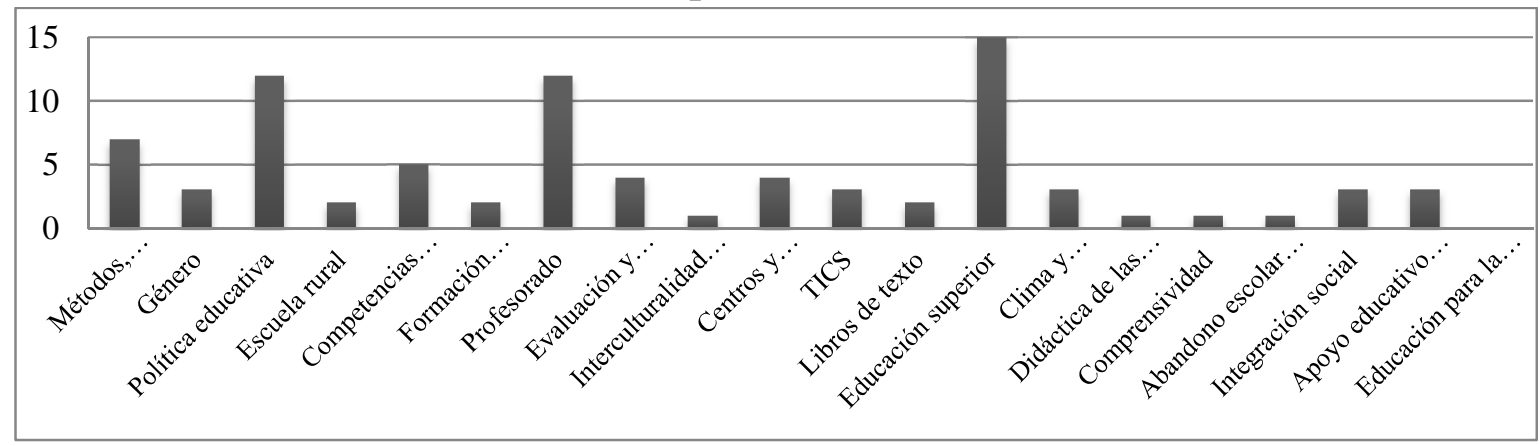

* Fuente: elaboración propia.

Las temáticas en el resto de revistas mantienen una relación parecida (Gráficos 7 10), siempre con la presencia de alguna de las tres más comunes aunque con un número de artículos mucho menor. Por ejemplo, como era de esperar, en la revista Profesorado predomina la temática "Profesorado", si bien es cierto que está muy condicionada por el monográfico sobre el profesorado de Música (recordemos que aportaba 10 artículos); así que, extrayendo ese monográfico, la temática "Profesorado" estaría al nivel del resto de 
temáticas presentes en los artículos analizados. Esta tendencia se repite para el resto de revistas que están centradas en un tema.

\section{Gráfico 7. Número de artículos por temática en la Revista Española de Pedagogía}

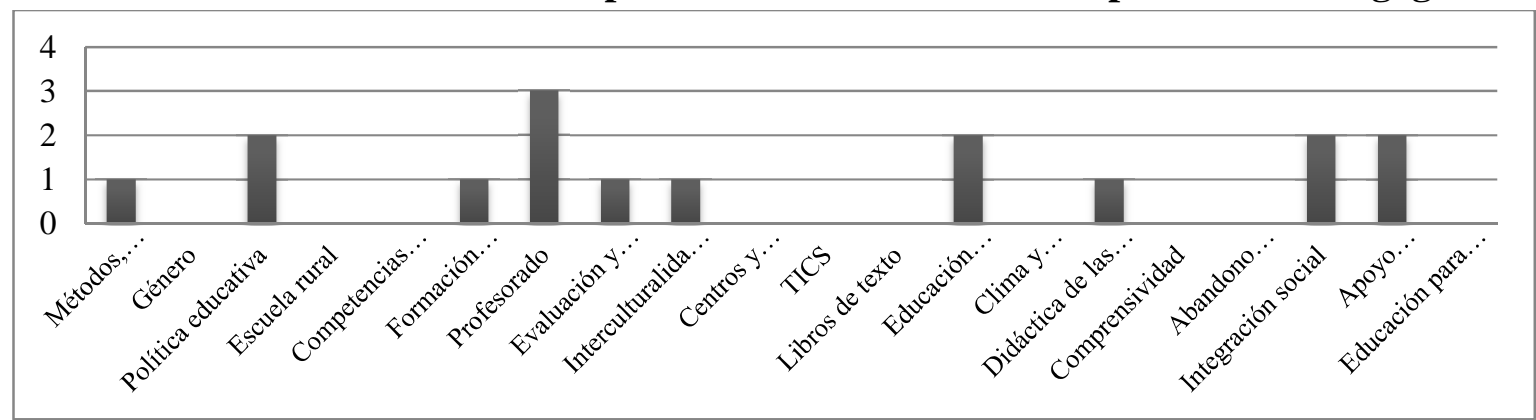

* Fuente: elaboración propia.

Gráfico 8. Número de artículos por temática en la Revista Educación XX1

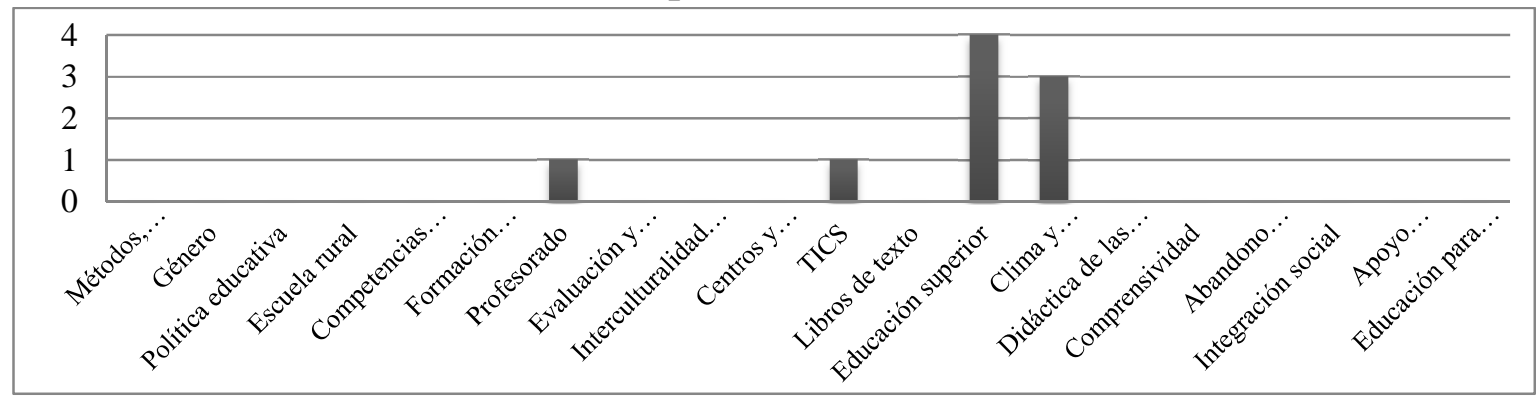

* Fuente: elaboración propia.

Gráfico 9. Número de artículos por temática en la Revista Profesorado

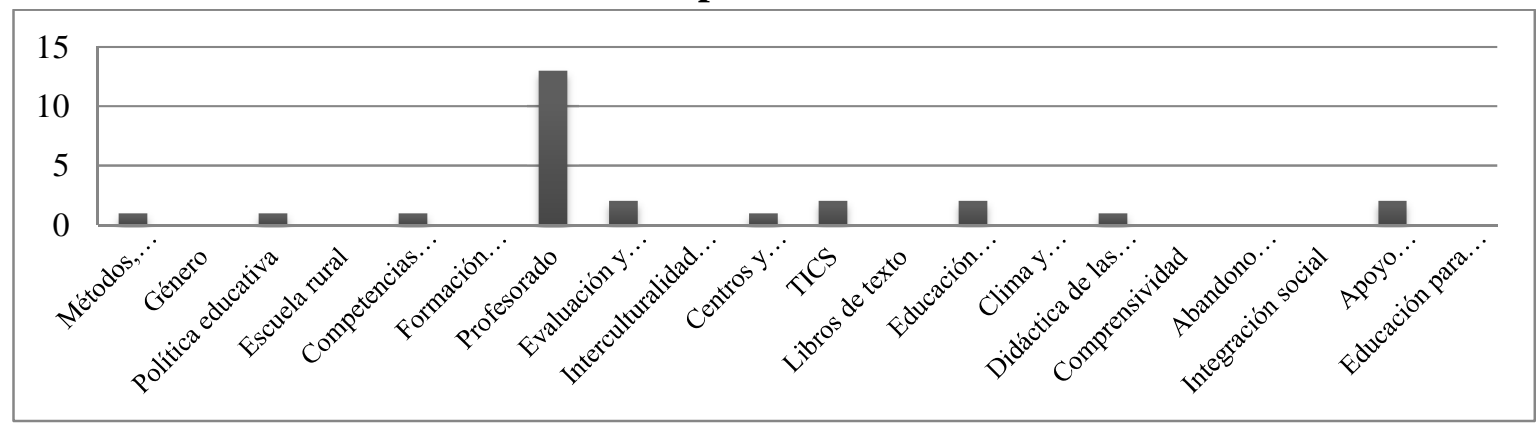

* Fuente: elaboración propia.

Gráfico 10. Número de artículos por temática en la Revista Bordón

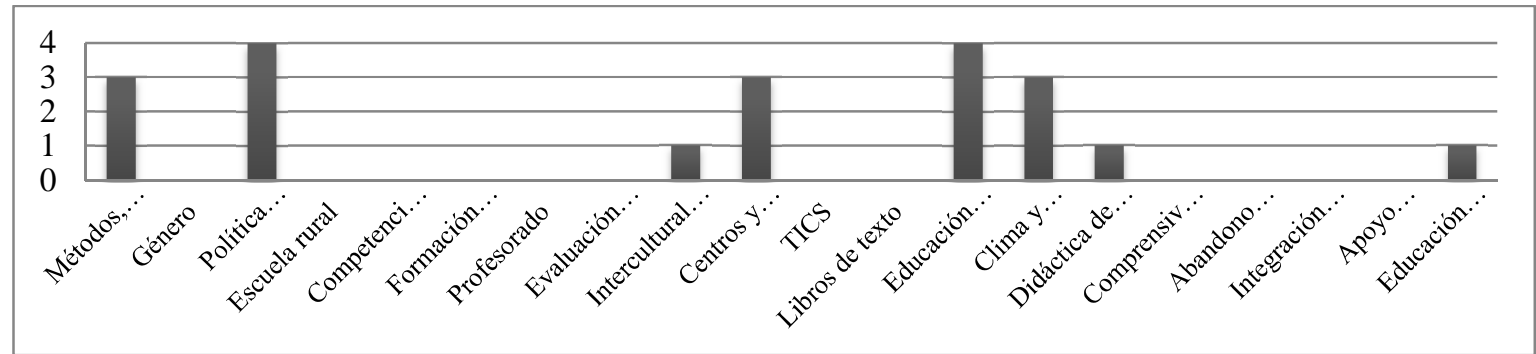

* Fuente: elaboración propia. 


\subsection{Citas}

Este subapartado seguramente sea el más interesante de cara al análisis de la producción científica. En esta investigación, la búsqueda de las citas se ha realizado a través de Google Scholar y no se ha establecido diferencias entre las citas totales recibidas, y las citas recibidas los tres primeros años desde su publicación. Se ha considerado que lo importante era conocer el impacto de los artículos en todo el periodo de años analizado y no tanto el impacto de la revista a la que pertenece.

Tabla 6. Número total de citas por revista

\begin{tabular}{|l|c|c|c|}
\hline Nombre de la revista & $\mathbf{N}^{\mathbf{o}}$ total de citas & x revista & x total \\
\hline Revista de Educación & 1026 & 12,21 & 6,62 \\
\hline Revista Profesorado & 128 & 4,92 & 0,82 \\
\hline Revista Española de Pedagogía & 93 & 5,81 & 0,60 \\
\hline Revista Educación XX1 & 40 & 4,44 & 0,25 \\
\hline Revista Bordón & 28 & 1,40 & 0,18 \\
\hline
\end{tabular}

* Fuente: elaboración propia.

Como en el resto del estudio, en este caso la Revista de Educación vuelve a estar desmarcada del resto (Gráfico 11). Los artículos publicados en ella reciben una cantidad de citas notablemente superior. Así, Revista de Educación tiene una media de 12,21 citas por artículo seleccionado y una media de 6,62 citas sobre el total de los 155 artículos analizados. Los casos de Revista Española de Pedagogía y Profesorado son llamativos, porque encontramos que Profesorado tiene un número total de citas superior al de Revista Española de Pedagogía, sin embargo, Revista Española de Pedagogía tiene una media de citas por artículo publicado en ella superior al de la revista Profesorado (5,81 frente a 4,92). Teniendo en cuenta el número medio de citas por revista sobre el total de artículos, la media final sería de 1,69 citas por artículo.

\section{Gráfico 11. Total de citas por revista}

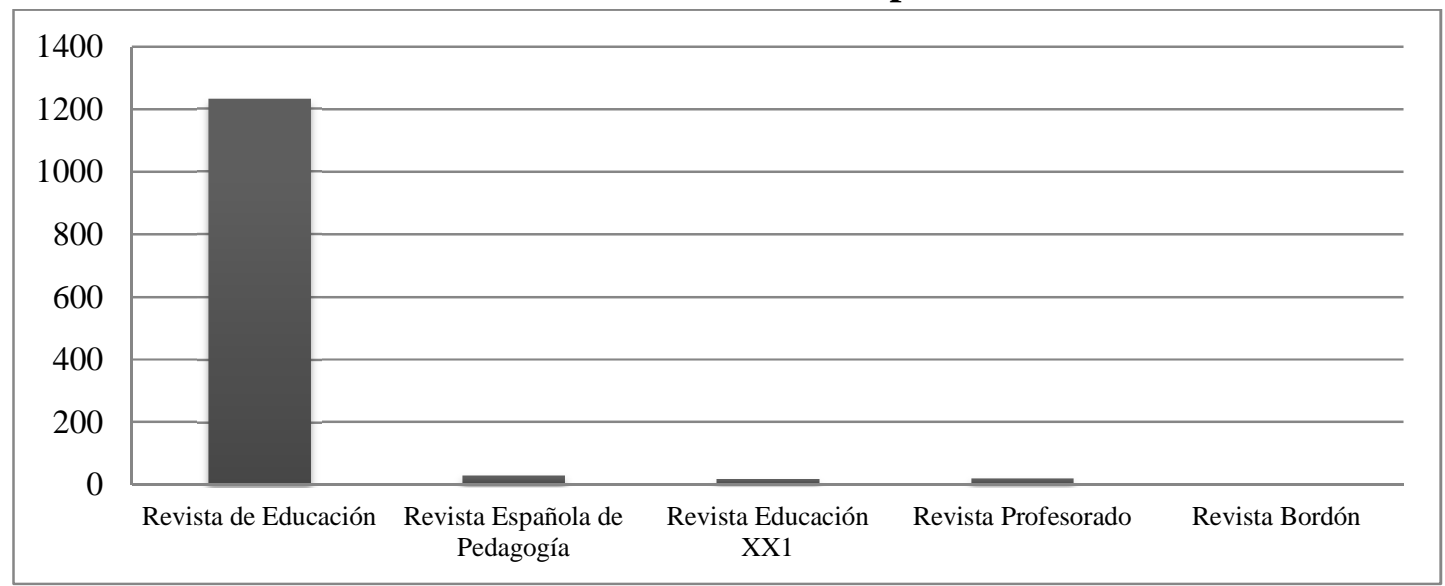

* Fuente: elaboración propia. 
Si observamos el número total de citas que recibieron los artículos seleccionados en cada uno de los años del periodo analizado, encontramos que los años con mayor éxito de impacto fueron 2006, 2004, 2008 y 2005. Estos datos no están directamente relacionados con los años en los que más artículos se publicaron (ver gráfico 2), por lo tanto indica que el mérito radica en los propios artículos y no en mero sumatorio de pocas citas en muchos artículos del mismo año. También es significativo los primeros y los últimos años de la muestra. En el caso de los primeros años, es destacable que con 6 artículos entre 1995 y 1996, hayan conseguido un total de 26 citas en prácticamente 20 años; además, estas 26 citas se deben en su práctica totalidad a un solo artículo que obtuvo 24. Por otro lado, los dos últimos años de la muestra con un total de 12 artículos han logrado 10 citas y todas son de los cuatro artículos del 2013. Esto último se explica porque apenas ha habido tiempo para que estos artículos sean citados por artículos posteriores; este es un hecho con el que se contaba al inicio del estudio y que no debería tenerse en cuenta.

Gráfico 12. Número de citas por años

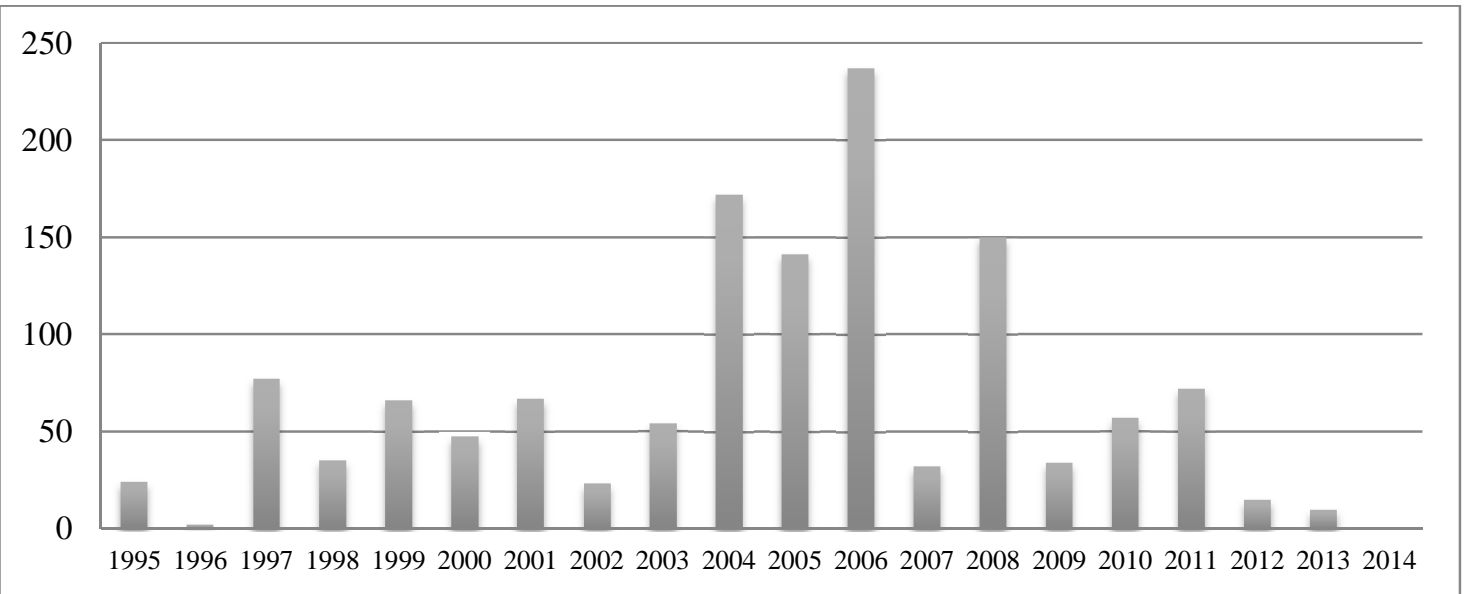

* Fuente: elaboración propia.

Si nos detenemos en los artículos que han recibido más número de citas (Tabla 7) encontramos en una posición muy adelantada el artículo de Rodríguez, Fita y Torrado (RODRÍGUEZ et al., 2004) sobre el rendimiento académico en la transición secundaria universidad en un estudio en el que se analizaron dos cohortes de estudiantes recién accedidos a la Universidad de Barcelona entre los años 1998 y 2000, que recibió un total de 65 citas. Por debajo de este tenemos 4 artículos con más 40 citas, 5 artículos con más de 30 citas, 11 artículos con más de 20 citas, 28 artículos con más de 10 citas y, por último, 106 artículos con menos de 10 citas. De este último grupo, únicamente 5 artículos son anteriores al año 2000, y 11 son posteriores al 2012; así pues, nos encontramos con 5 artículos que no han tenido ningún impacto en un margen de 15 - 20 años, y 11 que a penas han tenido tiempo para ser citados. Por lo tanto, tenemos 18 artículos que no han tenido ningún impacto, lo que significa el 11,6\% del total de artículos analizados. 
Tabla 7. Artículos con 10 citas o más ordenados decrecientemente

\begin{tabular}{|c|c|c|c|}
\hline REVISTA & AÑO & AUTORES & $\mathbf{N}^{\circ}$ CITAS \\
\hline Revista de Educación & 2004 & Rodríguez y otros & 65 \\
\hline Revista de Educación & 1999 & De Miguel y Arias & 48 \\
\hline Revista de Educación & 2006 & Blaya y otros & 47 \\
\hline Revista de Educación & 2006 & Fernández y otros & 43 \\
\hline Revista de Educación & 2005 & Sánchez y Zubillaga & 40 \\
\hline Revista de Educación & 2006 & Azcárate y Serradó & 32 \\
\hline Revista de Educación & 2003 & Mora & 31 \\
\hline Revista de Educación & 2011 & Brunner & 31 \\
\hline Profesorado & 2008 & Mulder y otros & 31 \\
\hline Revista de Educación & 1997 & Abraham y Rojas & 30 \\
\hline Revista de Educación & 2001 & Rodríguez & 28 \\
\hline Revista de Educación & 1997 & Calderhead & 25 \\
\hline Revista de Educación & 2004 & Caballero y Salvador & 25 \\
\hline Revista Española de Pedagogía & 2005 & Vez y Montero & 24 \\
\hline Revista de Educación & 1995 & Neave & 23 \\
\hline Revista Española de Pedagogía & 2008 & Pesquero y otros & 23 \\
\hline Revista de Educación & 2005 & Ortega & 22 \\
\hline Revista de Educación & 2008 & Docampo & 21 \\
\hline Revista de Educación & 2008 & Michavila y Zamorano & 21 \\
\hline Revista de Educación & 2006 & Vegas & 20 \\
\hline Educación XX1 & 2006 & Sanz & 20 \\
\hline Revista de Educación & 2006 & Pedró & 18 \\
\hline Revista de Educación & 2008 & Haug & 18 \\
\hline Revista de Educación & 2001 & Huguet & 17 \\
\hline Revista de Educación & 1998 & Fernández y Barquín & 16 \\
\hline Revista de Educación & 2000 & Sarramona & 16 \\
\hline Revista de Educación & 2004 & Pérez & 16 \\
\hline Revista de Educación & 2011 & Aramendi y otros & 16 \\
\hline Revista de Educación & 2003 & Villarroya & 15 \\
\hline Revista de Educación & 2004 & Ruiz y otros & 15 \\
\hline Revista de Educación & 2006 & Martínez & 15 \\
\hline Revista Española de Pedagogía & 2009 & Gairín y otros & 15 \\
\hline Revista de Educación & 1997 & Marcelo & 13 \\
\hline Revista de Educación & 1998 & Day & 13 \\
\hline Revista de Educación & 2001 & Mar-Molinero & 13 \\
\hline Revista de Educación & 2012 & López-Goñi y Goñi & 13 \\
\hline Revista de Educación & 2004 & Duru-Bellat & 12 \\
\hline Revista de Educación & 2005 & Sanz & 12 \\
\hline Revista de Educación & 2005 & Vega & 12 \\
\hline
\end{tabular}




\begin{tabular}{|l|c|l|c|}
\hline Profesorado & 2006 & Mulford & 12 \\
\hline Profesorado & 2006 & Ventura y otros & 12 \\
\hline Revista de Educación & 2000 & De la Fuente y Sánchez & 11 \\
\hline Revista de Educación & 2010 & Alegre y Benito & 11 \\
\hline Profesorado & 2005 & Grossman & 11 \\
\hline Revista de Educación & 1999 & Beduwe y Giret & 10 \\
\hline Revista de Educación & 2005 & De la Caba y López & 10 \\
\hline Revista de Educación & 2006 & Jares & 10 \\
\hline Revista de Educación & 2008 & Bruno-Jofré y Jover & 10 \\
\hline Revista Española de Pedagogía & 2010 & Ferrer y otros & 10 \\
\hline
\end{tabular}

* Fuente: elaboración propia.

\subsection{Geografía de la Educación Comparada}

En este último subapartado ubicaremos a cada autor en su institución de referencia y analizaremos la procedencia geográfica. Independientemente de quién fuera el autor principal, si se especificaban las instituciones de procedencia de todos los autores, se han tenido todas en cuenta. Para el caso de la ubicación geográfica se han tenido en cuenta los países, y en el caso español las Comunidades Autónomas, con el fin de hacer un análisis más pormenorizado de la geografía de la Educación Comparada en España. Estos datos no hablan de la nacionalidad de los autores, sino de los países desde los que han firmado las autorías de los artículos, es decir, el lugar de procedencia de las instituciones en las que trabajan. Así, nos encontramos, por ejemplo con el caso de Francesc Pedró de nacionalidad española, trabajando en la OCDE con domicilio en Paris (Francia), y se ha tenido en cuenta, para estos efectos, Francia y no España.

Gráfico 13. Tipos de organismos de procedencia de los autores

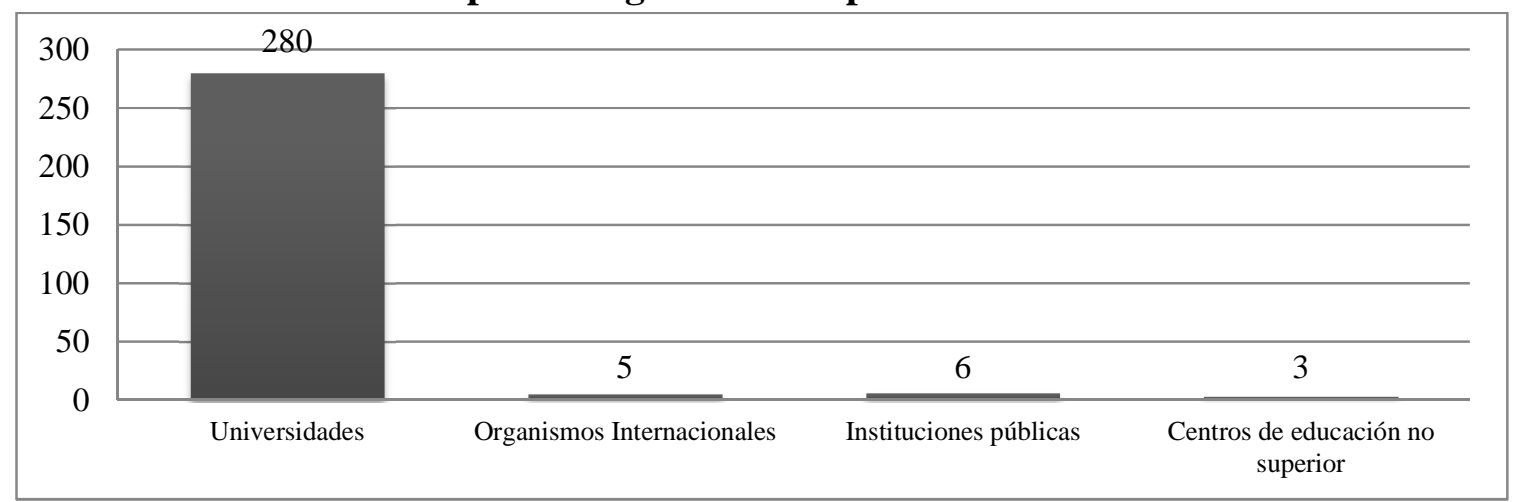

* Fuente: elaboración propia.

Como podemos observar en el Gráfico 13, las universidades son las grandes protagonistas muy por encima de las instituciones públicas (ministerios, administraciones centrales, servicios autonómicos, etc.), organismos internacionales y centros de educación 
no superior. Dentro de las universidades existe un nada desdeñable presencia del $18 \%$ de universidades extranjeras, lo que supone un total de 51 artículos de 39 universidades diferentes.

Si nos fijamos en los países de procedencia nos encontramos con una significativa presencia de 10 autores mexicanos, 9 estadounidenses, 8 franceses, 6 ingleses y 5 chilenos. En términos regionales - continentales tenemos 264 autores que pertenecen a instituciones localizadas en la Unión Europea, 17 en América Latina, 10 en América del Norte 2 en Asia, 1 en África y 1 en Oriente Medio.

Tabla 8. Geografía de los autores por países

\begin{tabular}{|c|c|}
\hline Países & Número de autorías \\
\hline España & 236 \\
\hline México & 10 \\
\hline EEUU & 9 \\
\hline Francia & 8 \\
\hline Reino Unido & 6 \\
\hline Chile & 5 \\
\hline Portugal & 4 \\
\hline Alemania & 4 \\
\hline Países Bajos & 2 \\
\hline Japón & 2 \\
\hline Suecia & 2 \\
\hline Israel & 1 \\
\hline Brasil & 1 \\
\hline Italia & 1 \\
\hline Argentina & 1 \\
\hline Finlandia & 1 \\
\hline Zimbabwe & 1 \\
\hline Canadá & 1 \\
\hline
\end{tabular}

* Fuente: elaboración propia.

Centrándonos al caso español (Mapa 1), tenemos que tener en cuenta dos aspectos que pueden resultar fundamentales a la hora de interpretar estos datos. Por un lado, se ha considerado a la Universidad Nacional de Educación a Distancia como una universidad localizada en la Comunidad Autónoma de Madrid a todos los efectos, sin tener en cuenta que es posible que los autores de estos artículos pertenezcan a dicha universidad, pero no estén en Madrid. Por otro lado, la revista Profesorado pertenece a la Universidad de Granada, concretamente es una revista impulsada por el grupo de investigación en Formación del Profesorado Centrada en la Escuela (FORCE - HUM-386), y por lo tanto, 
los datos geográficos están sobredimensionados para este caso porque la gran mayoría de artículos seleccionados de dicha revista han sido de autores de la propia universidad. Esto mismo ocurre con la revista Educación XX1 que es editada por la Facultad de Educación de la UNED.

Tabla 9. Geografía de los autores por Comunidades Autónomas.

\begin{tabular}{|c|c|}
\hline Comunidades Autónoma & Número de autorías \\
\hline Andalucía & 68 \\
\hline Catalunya & 37 \\
\hline Madrid & 37 \\
\hline País Vasco & 15 \\
\hline Administración Central & 14 \\
\hline Castilla y León & 13 \\
\hline Valencia & 13 \\
\hline Navarra & 10 \\
\hline Galicia & 10 \\
\hline Murcia & 4 \\
\hline Islas Canarias & 3 \\
\hline Sin especificar & 3 \\
\hline Asturias & 2 \\
\hline Castilla - La Mancha & 2 \\
\hline Extremadura & 2 \\
\hline Islas Baleares & 2 \\
\hline Aragón & 1 \\
\hline
\end{tabular}

* Fuente: elaboración propia.

Partiendo de estas premisas, la Comunidad Autónoma con mayor presencia es Andalucía con un total de 68 autorías, el 29\% del total, seguida muy de lejos por Catalunya y Madrid con 37 autorías cada una, el 16\% cada una sobre el total. Los casos de Cantabria, Ceuta, La Rioja o Melilla, sin ninguna representación, no son especialmente preocupantes porque a penas tienen presencia de universidades en su territorio. En el Mapa 1 se observa perfectamente como predominan las Comunidades Autónomas de Andalucía, Madrid y Catalunya siendo estas también las que más universidades albergan en su territorio. 
Mapa 1. Geografía de los autores por Universidades

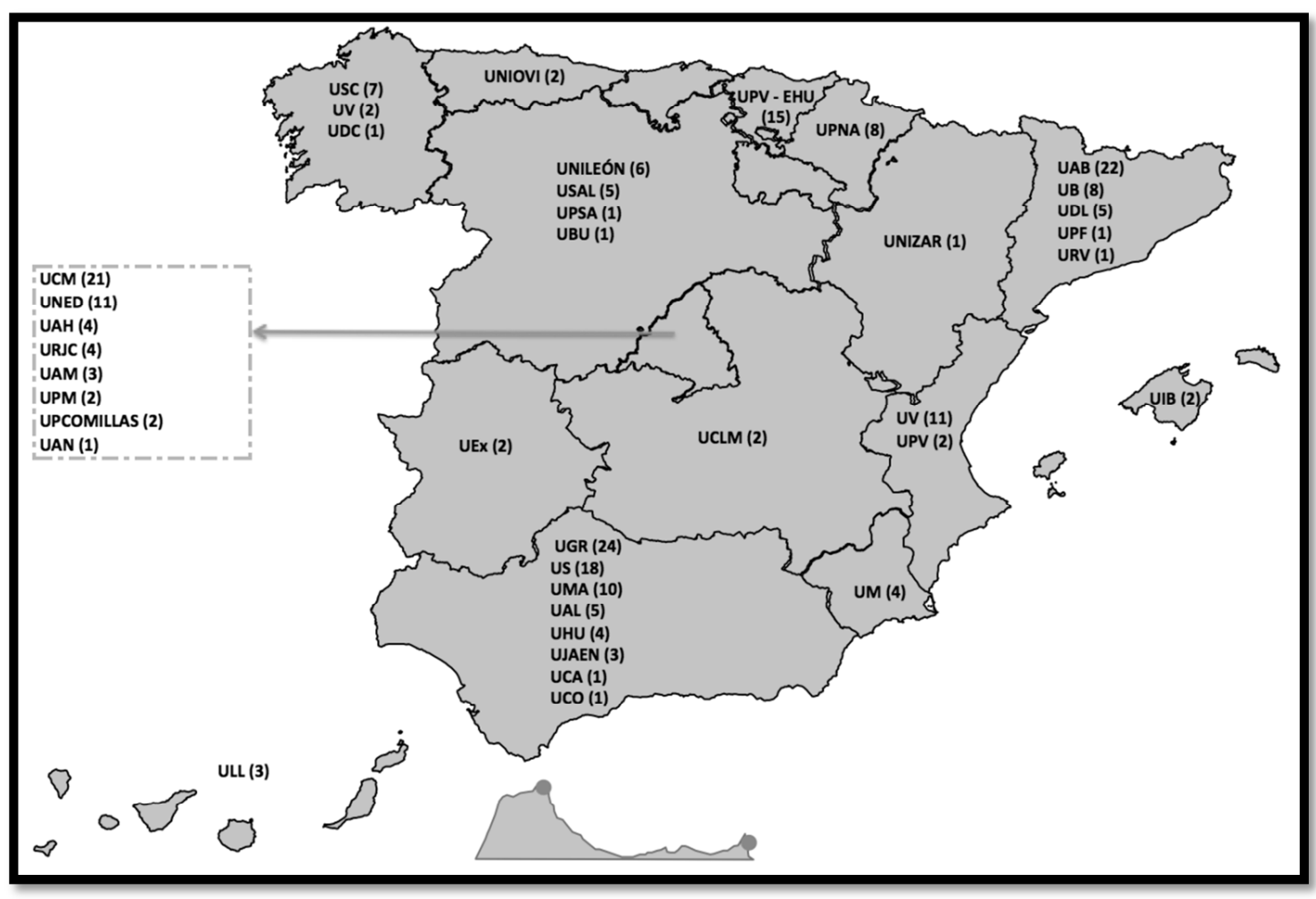

* Fuente: elaboración propia.

Por último, en cuanto al número de autorías, encontramos a la Universidad de Granada con el $10 \%$ de las autorías españolas y con el $8 \%$ de las autorías sobre el total de universidades. Seguida muy de cerca por la Universidad Autónoma de Barcelona con el 9\% y el $7 \%$ respectivamente. En tercer lugar encontramos a la Universidad Complutense de Madrid con el 8,9\% de las autorías españolas y el 7,5\% de las autorías sobre el total de universidades. Sólo encontramos dos universidades extranjeras con 3 o más autorías, las dos mexicanas, la Universidad Autónoma de Sinaloa y la Universidad Autónoma de Yucatán.

Tabla 10. Universidades con 3 o más autorías

\begin{tabular}{|c|c|}
\hline Nombre de la Universidad & Número de autorías \\
\hline Universidad de Granada & 24 \\
\hline Universidad Autónoma de Barcelona & 22 \\
\hline Universidad Complutense de Madrid & 21 \\
\hline Universidad de Sevilla & 18 \\
\hline Universidad del País Vasco & 15 \\
\hline Universidad Nacional de Educación a Distancia & 11 \\
\hline Universidad de Valencia & 11 \\
\hline Universidad de Málaga & 10 \\
\hline
\end{tabular}




\begin{tabular}{|c|c|}
\hline Universidad de Barcelona & 8 \\
\hline Universidad Pública de Navarra & 8 \\
\hline Universidad de Santiago de Compostela & 7 \\
\hline Universidad de León & 6 \\
\hline Universidad de Almería & 5 \\
\hline Universidad de Lleida & 5 \\
\hline Universidad de Salamanca & 5 \\
\hline Universidad de Alcalá & 4 \\
\hline Universidad de Aveiro & 4 \\
\hline Universidad de Huelva & 4 \\
\hline Universidad de Murcia & 4 \\
\hline Universidad Rey Juan Carlos & 3 \\
\hline Universidad Autónoma de Madrid & 3 \\
\hline Universidad Autónoma de Sinaloa & 3 \\
\hline Universidad Autónoma de Yucatán & 3 \\
\hline Universidad de Jaén & 3 \\
\hline Universidad de La Laguna & \\
\hline & \\
\hline
\end{tabular}

* Fuente: elaboración propia.

\section{CONCLUSIONES Y DISCUSIÓN}

El estudio biblométrico que se acaba de presentar nos permite identificar algunas fortalezas y debilidades en torno a las publicaciones que la disciplina de la Educación Comparada realiza en España, sin que estas pretendan ser las únicas ni las que mejor definan a la disciplina.

En primer lugar, antes de entrar a discutir estas fortalezas y las debilidades de la producción científica educativa y comparada en España, es importante señalar que el hecho de que la selección de las revistas se haya hecho en base a los índices de impacto solo pretende aportar valor añadido a los artículos, sin menospreciar el trabajo de otras revistas y otros investigadores, pues la calidad no reside únicamente en el índice de impacto.

En una primera visión general encontramos dos limitaciones en el objeto de estudio. Por una parte es identificable el sesgo en la selección de artículos. Tanto la revista Educación XX1, como la revista Profesorado. Revista de currículum y formación del profesorado, publican más artículos de investigadores relacionados con la universidad propulsora de la revista; este hecho también ha sido identificado por otro estudio (GÓMEZGARCÍA et al. 2012) para el caso de Educación XX1. Sin embargo, como nos apuntan Fuentes y otros (2012: 212) "debido al aún reducido número de opciones para publicar en alguna de las revistas incluidas en el JCR de habla hispana, el criterio endogámico debe ser relativizado". Por lo tanto, no debemos minusvalorar el trabajo de los investigadores y académicos de estas universidades por el mero hecho de publicar ellas, ya que deben 
someterse igualmente a los criterios de selección de los artículos y a la revisión por pares ciegos. Por otra parte existe una supremacía del español como idioma de publicación, que se puede interpretar como un elemento limitador del impacto de estos artículos. Para poder extraer una conclusión más rigurosa al respecto hubiera sido necesario extraer la media de citas por idioma utilizado y haber ampliado el seguimiento a artículos en revistas extranjeras de autores españoles, así que tan solo se puede apuntar esta hipótesis.

En cuanto a las autorías es significativo que más de la mitad de los artículos publicados se hayan hecho en solitario, esto pone de manifiesto la soledad del comparatista. Esta soledad viene acompañada de una mayor presencia de artículos teóricos que empíricos cuantitativos, para los cuales es necesario una mayor participación de autores dado el tratamiento estadístico necesario, en contraposición, con la necesidad del análisis y reflexión individual de los trabajos de corte más cualitativo o descriptivos propios de la Educación Comparada.

En el apartado de las temáticas ya hemos puesto de manifiesto en los resultados que existe una preponderancia de las temáticas de Educación Superior, Profesorado y Política Educativa, siendo las tres temáticas claramente identificables con la disciplina en cuestión; pero además, estos resultados, escenifican la gran variedad de temáticas que en la práctica son tratadas a través de la metodología comparada. Este aspecto nos da una idea de la utilidad de esta metodología y de la salud que goza en el mundo académico - investigador.

Sin embargo, en contraposición con la situación que se puede extraer de las temáticas, el número de citas nos sitúa en un lugar mucho más humilde de lo que hasta ahora podría parecer. Según las revistas analizadas, la media final de citas por artículo hemos encontrado que es de 1,69, que está lejos de ser un impacto relevante. Hubiese sido interesante comparar las medias del número de citas de los artículos seleccionados con las medias del número de citas de cada revista, pero estos datos no están disponibles y los que están disponibles son de años anteriores, con lo que el resultado estaría distorsionado. Así que, a partir de los datos de los que disponemos, una media de 1,69 citas por artículo parece insuficiente dada la media de citas de Revista de Educación en este estudio $(6,62)$, aunque es cierto que está por encima de la media del resto de revistas.

Por último, la geografía de la educación comparada nos muestra un panorama concentrado en los grandes núcleos geográficos universitarios en cuanto al volumen de artículos publicados se refiere. El protagonismo de las universidades es tal que se puede interpretar de dos formas diferentes (FUENTES et al., 2012): la investigación comparada está reducida a la universidad, y/o existe lejanía entre la investigación y la práctica educativa real, es decir, entre las investigaciones y las aulas. Sea como fuere, la presencia de otros organismos o instituciones no universitarias es muy reducido en el campo de la metodología comparada. Quizás debamos reflexionar sobre esto y acercar la disciplina (y 
sobre todo su utilidad para el desempeño docente) a la práctica real de las escuelas. Si bien es cierto que si relacionamos este hecho con las temáticas, podemos encontrarnos con que la investigación comparada no se centra en los problemas cotidianos de las aulas, sino que está más cerca de ser de utilidad a los policymakers.

Antes de finalizar, quisiéramos señalar que hubiese sido interesante ampliar el estudio bibliométrico hacia aspectos que nos permitieran conocer los artículos con metodología comparada se han quedado fuera en los procesos de aceptación de artículos de las revistas, o quizás otros aspectos como la financiación de los estudios o las metodologías utilizadas en los artículos publicados.

En definitiva, a través de este estudio hemos puesto de relieve que el método comparado, y por analogía la Educación Comparada, goza de un estado relativamente saludable. Presente en las revistas de mayor impacto científico del país, cercana a los grandes núcleos universitarios y con un impacto cada vez mayor, estamos ante una disciplina que sigue en auge y que se consolida en el panorama nacional español.

\section{REFERENCIAS BIBLIOGRÁFICAS}

ANECA (2007): Programa de Evaluación del Profesorado para la Contratación. Principios y orientaciones para la aplicación de los criterios de evaluación. (ANECA, Madrid).

ANECA (2008): Programa ACADEMIA. Principios y orientaciones para la aplicación de los criterios de evaluación. (ANECA, Madrid).

CARPALlO, A. y BURGOS, E. (2008): Estudio bibliométrico y de calidad de la Revista Complutense de Educación (1990-2007). Revista Complutense de Educación, 19 (1), pp. 13-29.

DELGADO, E., RUIZ-PÉREZ, R., y JIMÉNEZ-CONTRERAS, E. (2006): La Edición de Revistas Científicas Directrices, Criterios y Modelos de Evaluación. (EC3 Universidad de Granada, Granada).

EGIDO, I. (2014): Veinte años de Educación Comparada en España: un repaso a las publicaciones del periodo 1994 - 2014. Revista Española de Educación Comparada, 24, pp. 125-151.

FERNÁNDEZ LAMARRA, N., MOLLIS, M. y DONO, S. (2005): La Educación Comparada en América Latina: situación y desafíos para su consolidación académica. Revista Española de Educación Comparada, 11, pp. 161-187.

FUENTES, J.L., LUQUE, D. y LÓPEZ, E. (2012): Análisis bibliométrico de las revistas españolas de educación incluidas en el Journal Citation Report. Producción científica y elementos controvertidos. Teoría de la Educación. Revista Interuniversitaria, 24, pp. 183-217. 
GARCÍA, M. J. (2012): Impacto de la globalización y el postmodernismo en la epistemología de la Educación Comparada. Revista Española de Educación Comparada, 20, pp. 41-80.

GARCÍA GARRIDO, J.L. (1996): Fundamentos de Educación Comparada. ( $3^{\text {a }}$ ed.) (Madrid, Dykinson).

GARCÍA GARRIDO, J.L. (2005): Diez años de Educación Comparada en España. Revista Española de Educación Comparada, 11, pp. 15-36.

GÓMEZ-GARCÍA, A., RAMIRO, M.T., ARIZA, T. y GRANADOS, M. R. (2012): Estudio bibliométrico de Educación XX1, Educación XX1, 15 (1), pp. 17-41.

GRANADOS, M.R., ARIZA, T., GÓMEZ-GARCÍA, A., y RAMIRO, M.T. (2011): Estudio bibliométrico de Aula Abierta. Aula Abierta, 39 (3), pp. 97-110.

LÁZARO, J.M., LORENTE, M. y ANCHETA, A. (2014): Cartografía académica de la Educación Comparada en la universidad española actual. Revista Española de Educación Comparada, 24, pp. 153-171.

LÁZARO, J.M. (2013): Comparative and international education in Spain: Current approaches and directions for future teaching and research. International Perspectiva of Education and Society, 20, pp. 37-42.

LÓPEZ, A. (2008): Retos metodológicos de la educación comparada en la sociedad global. Revista de Universidad y Sociedad del Conocimiento, 5 (1), pp. 1-9.

LÓPEZ, E., VÁZQUEZ, E. y SARASOLA, J.L. (2015): Estudio bibliométrico de Pixel-Bit, Revista de Medios y Educación (2000-2013). Píxel-Bit, Revista de Medios y Educación, 46, pp. 65-85.

MARTÍNEZ, M.J. y VALLE, J.M. (2005): 10 años de la REEC. Una mirada en perspectiva. Revista Española de Educación Comparada, 11, 37-93.

MONTERO, I. y LEÓN, O.G. (2007): A guide for naming research studies in Psychology. International Journal of Clinical and Health Psychology, 7 (3), pp. 847-862.

NAYA, L. M. (2005): La Educación Comparada en los nuevos espacios virtuales (19952004). Revista Española de Educación Comparada, 11, pp. 241-271.

NÍKLEVA, D. G. y CORTINA, B. (2014): La producción científica actual en Didáctica de la Lengua y la Literatura en revistas españolas. Porta Linguarum, 21, pp. 281-299.

RAMOS-ÁLVAREZ, M. M., MORENO-FERNÁNDEZ, M. M., VALDÉZ-CONRO, B. y CATENA, A. (2008): Criteria of the peer review process for publication of experimental and quasi-experimental research in Psychology: A guide for creating research papers. International Journal of Clinical and Health Psychology, 8, pp. 751764.

RAVENTÓS, F. y GARCÍA, M. J. (2012): Educación Comparada, Globalización y Postmodernismo. Revista Española de Educación Comparada, 20, pp. 13-18.

RAVENTÓS, F. y PRATS, E. (2012): Sociedad del conocimiento y globalización. Nuevos retos para la Educación Comparada. Revista Española de Educación Comparada, 20, pp. 19-40. 
RODRÍGUEZ, S., FITA, E. y TORRADO, M. (2004): El rendimiento académico en la transición secundaria - universidad. Revista de Educación, 334, pp. 391-414

RUIZ-CORBELLA, M., GALÁN, A. y DIESTRO, A. (2014): Las revistas científicas de Educación en España: evolución y perspectivas de futuro. RELIEVE, 20, DOI: 10.7203/relieve.20.2.4361

RUIZ-PÉREZ, R., DELGADO, E. y JIMÉNEZ-CONTRERAS, E. (2010): Principios y criterios utilizados en España por la Comisión Nacional Evaluadora de la Actividad Investigadora (CNEAI) para la valoración de las publicaciones científicas: 19892009. Psicothema, 22, pp. 898-908.

TORRES-SALINAS, D., BORDONS, M., GIMÉNEZ-TOLEDO, E., DELGADO, E., JIMÉNEZ-CONTRERAS, E. y SANZ- CASADO, E. (2010): Clasificación integrada de revistas científicas (CIRC): propuesta de categorización de las revistas en ciencias sociales y humanas. El Profesional de la Información, 19(6), pp.675683.

ZYCH, I. (2011): Análisis bibliométrico de la Revista "Bordón”. Bordón, 63 (1), pp. 141152.

\section{PROFESIOGRAFÍA}

\section{Gabriel Álvarez López.}

Es Licenciado en Pedagogía en la Universidad de Salamanca en el año 2009. Realizó el Máster en Acción Solidaria Internacional de Europa en la Universidad Carlos III de Madrid en el año 2010. En el curso 2012-2013, en la Universidad Autónoma de Madrid realizó el Master en Calidad y Mejora de la Educación. Ese mismo año, empezó el Máster en Políticas Públicas y Sociales por la Universitat Pompeu Fabra de Barcelona y la John Hopkins University (Baltimore, EEUU), que está finalizando. Actualmente, es Personal Investigador en Formación en la Universidad Autónoma de Madrid donde está realizando el Doctorado con una tesis doctoral titulada "La evaluación de la Educación Básica en las Comunidades Autónomas, desde una perspectiva supranacional: estudio comparado y de casos", en el Departamento de Didáctica y Teoría de la Educación, dirigida por Javier M. Valle. Datos de contacto: Email: gabriel.alvarez@estudiante.uam.es.

Fecha de recepción: 25 de marzo de 2015.

Fecha de aceptación: 6 de mayo de 2015 . 